Revue musicale OICRM

\title{
(Re)présenter les œuvres musicales. L'exemple des programmes de concert de la Société de musique contemporaine du Québec
}

\section{Ariane Couture}

Volume 2, numéro 2, 2015

Une relève

URI : https://id.erudit.org/iderudit/1060130ar

DOI : https://doi.org/10.7202/1060130ar

Aller au sommaire du numéro

Éditeur(s)

OICRM

ISSN

2368-7061 (numérique)

Découvrir la revue

Citer cet article

Couture, A. (2015). (Re)présenter les œuvres musicales. L'exemple des programmes de concert de la Société de musique contemporaine du Québec. Revue musicale OICRM, 2(2), 44-66. https://doi.org/10.7202/1060130ar

\section{Résumé de l'article}

À partir du concept de paratexte développé par Genette (1987) et adapté à la musique par Escal (1996), cet article met au jour les modalités de (re)présentation des œuvres jouées par la Société de musique contemporaine du Québec (SMCQ) telles que véhiculées par les programmes de concerts produits entre 1966 et 2013. Après avoir effectué une mise en contexte de l'historique, du fonctionnement et de la programmation des œuvres de la SMCQ, les programmes de concerts sont examinés dans une perspective comparativiste afin d'en faire ressortir les similarités et les divergences. En premier lieu, la description formelle de la page couverture, du format et de la mise en page des programmes dirige la réflexion sur les paratextes non verbaux. En second lieu, l'examen des notices écrites des œuvres les plus jouées de la SMCQ permet d'affirmer l'importance accordée à l'autorité des compositeurs, principaux auteurs des paratextes verbaux. Ainsi, l'article permet d'éclairer l'articulation et l'évolution de la pensée musicale de la SMCQ pendant cette période. 


\title{
(Re)présenter les œuvres musicales. L'exemple des programmes de concert de la Société de musique contemporaine du Québec
}

\author{
Ariane Couture
}

\begin{abstract}
Résumé
À partir du concept de paratexte développé par Genette (1987) et adapté à la musique par Escal (1996), cet article met au jour les modalités de (re)présentation des œuvres jouées par la Société de musique contemporaine du Québec (SMCQ) telles que véhiculées par les programmes de concerts produits entre 1966 et 2013. Après avoir effectué une mise en contexte de l'historique, du fonctionnement et de la programmation des œuvres de la SMCQ, les programmes de concerts sont examinés dans une perspective comparativiste afin d'en faire ressortir les similarités et les divergences. En premier lieu, la description formelle de la page couverture, du format et de la mise en page des programmes dirige la réflexion sur les paratextes non verbaux. En second lieu, l'examen des notices écrites des œuvres les plus jouées de la sMce permet d'affirmer l'importance accordée à l'autorité des compositeurs, principaux auteurs des paratextes verbaux. Ainsi, l'article permet d'éclairer l'articulation et l'évolution de la pensée musicale de la sMce pendant cette période.
\end{abstract}

Mots clés: dicours sur la musique ; paratextualité ; programmes de concert ; représentation; Société de musique contemporaine du Québec.

\begin{abstract}
From the concept of paratext developed by Genette (1987) and adapted to music by Escal (1996), this article provides insights on the modalities of (re)presentation of works played by Société de musique contemporaine du Québec (sMce) as conveyed by concert's programs produced between 1966 and 2013. Following historical, organizational and musical programming contexts, concert's programs are studied in a comparativist perspective to show similarities and differences. First of all, formal descriptions of the front page, format and layout guide the reflection on non verbal paratexts. Second, examination on written notices of SMCQ's most played works asserts the authority given to composers, principal authors of the verbal paratexts about their own pieces. Thus, this article sheds light on SMCQ's musical thoughts' articulation and evolution during this period.
\end{abstract}

Keywords: concert programs; discourse about music; paratextuality; representation; Société de musique contemporaine du Québec. 


\section{LA CONSTITUTION D'UN CHAMP DE RECHERCHE SUR LE PROGRAMME DE CONCERT}

Lus avec attention, compulsés distraitement, consultés machinalement, triturés, pliés, roulés, les documents d'accompagnement des concerts, produits en quantité par les organismes musicaux, survivent rarement au cadre immédiat du concert, sauf pour quelques collectionneurs qui retrouvent dans ces objets anodins - livret de concert, affichette, talon de billet - le souvenir d'une expérience esthétique. Malgré l'utilité évidente de ces imprimés au moment du concert, ils sont le plus souvent ignorés, voir banalisés, par les musicologues au profit d'autres sources de documentation approuvées par la communauté scientifique, telles les partitions, les enregistrements sonores, les écrits de musiciens, ou encore les critiques de concert publiées dans la presse musicale. Quelques travaux récents s'appuient toutefois sur ces médias pour retracer l'histoire de la production et de la promotion de la musique de tradition occidentale savante.

Le premier destinataire du programme de concert étant l'auditeur (Simeone 2012), il n'est pas surprenant que la majorité des travaux y étant consacrés portent sur la réception des œuvres et les habitudes d'écoutes des auditeurs au XIX ${ }^{\mathrm{e}}$ siècle et au début du Xx ${ }^{e}$ siècle (Bashford 2003, Bent 1994, Botstein 1992, Campos et Donin 2005, Dale 2003). Par exemple, Campos et Donin (2005), dans une étude portant sur un florilège de guides d'écoute rédigés par les soins de Charles Malherbe, d'Étienne Destranges ou de Julien Tiersot, révèlent les stratégies d'écriture des musicographes en prise avec, d'un côté, les contraintes éditoriales liées à la publication de leurs textes et, de l'autre côté, l'autorité du compositeur sur son œuvre. L'analyse des guides d'écoute permet alors aux chercheurs de mettre en parallèle ces prescriptions sur les manières d'écouter la musique avec les pratiques auditives effectives du public : "L'espace public de l'écoute est en effet façonné par des routines collectives et par l'expression d'interventions singulières, tout en étant tramé par un espace public de l'analyse qui fournit un vocabulaire, des attentes et émets des suggestions " (Campos et Donin 2005 , p. 203). De manière générale, l'étude du programme de concert permettrait donc de mieux comprendre l'histoire de la réception des œuvres telle que relatée dans la presse musicale ou autres écrits de musiciens.

Dans un autre ordre d'idées, Jane Pasler (2007) utilise les programmes de divers ensembles musicaux parisiens entre 1890 et 1914 pour éclairer les valeurs que la société française a associées à la musique et les changements de valeurs opérés au fil du temps. Le graphisme de la page couverture, le format des caractères d'imprimerie, les publicités sont autant d'éléments analysés par l'auteure pour documenter l'évolution des goûts musicaux des Français d'une forme de modernisme associée à la féminité et à l'art nouveau, pour une autre, plus masculine, abstraite et fondée sur l'innovation (Pasler 2007, p. 365). De même, à partir des textes de présentation des compositeurs dans les notes de programme du Domaine musical, Nicolas Donin révèle que «le discours biographique pouvait donc tout à fait être mobilisé pour vulgariser, distiller des périodisations et une conception de l'histoire, enrôler la morale dans l'esthétique, (in)former le jugement de goût, orienter l'écoute " (Donin 2011, p. 47).

Le champ de recherche sur le programme de concert interroge donc l'emploi de ces imprimés éphémère - du guide de formation à l'appréciation esthétique des œuvres 
au véhicule de la signification globale de la musique dans une société donnée - sans perdre de vue que la manière dont le discours sur les œuvres se construit est fondé sur les habiletés et les attentes supposées des différents auditeurs visés.

Afin d'élargir le propos des travaux susmentionnés, les programmes de concerts produits entre 1966 et 2013 par la Société de musique contemporaine du Québec (SMCQ) seront considérés en tant que paratexte des œuvres diffusées ${ }^{1}$. Théorisé par Genette, le paratexte est une production verbale ou non qui accompagne un écrit littéraire et qui en propose une lecture. L'ensemble des messages paratextuels, qu'il soit assigné à l'auteur, l'éditeur ou un tiers, a donc deux fonctions solidaires, soit celle d'informer et celle de persuader (Escal 1996, p. ix). Certains éléments factuels et éditoriaux tels le nom d'auteur, le titre, le sous-titre, la table des matières, les références bibliographiques, servent à présenter une œuvre d'art. D'autres éléments, dont la préface et les notes infrapaginales, commentent l'œuvre et influencent sa réception.

Après une mise en contexte de l'historique, du fonctionnement et de la programmation des œuvres de la SMCQ, l'étude particulière des programmes de concert de la SMCQ se concentra sur quelques éléments inhérents aux paratextes musicaux décrits par Escal (1996)² pour éclairer les (re)présentations de la musique contemporaine au Québec. Ainsi, l'analyse portera, dans un premier temps, sur l'aspect visuel des programmes - plus précisément sur la page couverture, le format d'impression, l'ordonnancement des informations factuelles - afin d'en fournir une description physique et d'en retracer les similitudes et les changements opérés au fil du temps. Dans un second temps, ce sont les notes sur les œuvres musicales qui seront discutées pour comprendre les contextes de signification des programmes de la SMCQ. Cette analyse révélera le rôle important de la sMce quant à l'affirmation de l'autorité des compositeurs en utilisant leurs textes descriptifs et en rééditant les mêmes notices pour différents concerts. Ceci manifeste également une certaine volonté de standardisation du discours sur les œuvres.

\section{Les CONCERTS DE LA SociÉTÉ DE MUSIQUe CONTEMPORAINE DU QuÉBEC}

Fondée en 1966 à Montréal par Hugh Davidson (1930-2014), Serge Garant (1929-1986), Maryvonne Kendergi (1915-2011), Jean Papineau-Couture (1916-2000) et Wilfrid Pelletier (1896-1982), la Société de musique contemporaine du Québec est

\footnotetext{
1 Je remercie Serge Lacasse de m'avoir suggéré cet angle d'approche des programmes de concert suite à une conférence prononcée au 81 ${ }^{\mathrm{e}}$ congrès de l'Acfas en mai 2013 et de m'avoir par la suite référée aux ouvrages de Françoise Escal (1996) et de Gérard Genette (1987).

2 Le statut ontologique particulier des œuvres musicales a incité Escal (1996) à adapter la méthode d'analyse des éléments paratextuels de Genette. Si en littérature, la majorité des paratextes sont de même nature que le texte lui-même, en ce sens qu'il s'agit également de dispositifs écrits (à l'exception des entrevues radiophoniques ou télévisées), en musique, les objets d'accompagnement sont tributaires de la double nature des œuvres musicales : écrite (partition) et sonore (exécution, enregistrement). Pour rendre compte le plus largement possible des paratextes musicaux, Escal distingue donc les messages non verbaux (prologue et ouverture d'opéra, programmation, iconographie, corps du musicien, salle de concert) et les messages verbaux (nom de l'auteur, dédicace, notice d'œuvre, conférence pré-concert, entretien, etc.).
} 
le premier organisme québécois permanent dédié à la musique contemporaine. Sa direction artistique est assumée successivement par les compositeurs Serge Garant (1966-1986), Gilles Tremblay (1986-1988) et Walter Boudreau (depuis 1988). Le fonctionnement quotidien et la production des activités de la SMCQ sont confiés à un conseil d'administration, une direction administrative, une équipe de production, et un comité des programmes qui travaille en étroite collaboration avec le directeur artistique. Le financement de la SMCQ provient principalement de subventions gouvernementales (Conseil des arts de Montréal, Conseil des arts et lettres du Québec, Conseil des Arts du Canada), ainsi que de la vente de billets, d'ententes de service, de commandites et de dons.

En plus de quarante ans d'activités, la SMCQ a donné environ 820 représentations de concerts au Québec, mais également lors de tournées au Canada, aux États-Unis ou en Europe. En plus de produire les concerts de l'Ensemble de la sMCQ, l'organisme diffuse les représentations de musiciens locaux (par exemple : Ensemble Transmission, Quatuor Bozzini, Quatuor Molinari, Quatuor Quasar, Sixtrum) et reçoit des ensembles ou des solistes de réputation internationale tels l'Ensemble Intercontemporain dirigé par Pierre Boulez, les Percussions de Strasbourg, le Collegium vocal Köln sous la direction de Stockhausen ou plus récemment les Neue Volcalsolisten Stuttgart. Depuis 1997, la SMCQ développe un volet jeunesse dont les contes musicaux ont été présentés devant plus de 50000 enfants. Enfin, depuis 2003, la SMCQ organise le festival international Montréal/Nouvelles Musiques (MNM) sur une base bisannuelle. En alternance avec MNM, l'organisme tient une série hommage dédiée à un compositeur québécois et à laquelle participe de nombreux interprètes et ensembles en intégrant une œuvre de ce compositeur à leur programmation.

La programmation des concerts de la SMCQ reflète une volonté de développer un répertoire de musique contemporaine québécois sur la base de deux axes principaux : les œuvres canoniques $\mathrm{du} \mathrm{xx}^{\mathrm{e}}$ siècle et les œuvres présentées en première audition, les deux se répondant au sein d'un même concert ${ }^{3}$. Ce principe régit l'organisation structurelle et la mise en valeur des œuvres, lesquelles s'inscrivent, par association, dans une perspective historique. Citons à ce propos Serge Garant qui commente la première saison de la SMCQ :

Nous avons joué cette année deux classiques de la musique contemporaine : Varèse et Webern; quatre brillants représentants de la jeune musique, Boulez, Cage, Kagel et Stockhausen, ainsi que six compositeurs canadiens : Mather, Morel, Schafer, Somers, Tremblay, et moi-même. Nous entendons continuer dans le même sens : donner à la fois des classiques, des jeunes qui marquent la musique ailleurs, et des compositeurs d'ici (Garant 1967, p. 1).

3 Pour une analyse approfondie de la programmation musicale de la sMce, voir Couture (2013a). On retrouve également une politique de programmation semblable chez d'autres organismes spécialisés en musique contemporaine notamment, le Domaine musical en France (Aguila 1992) et le Nouvel Ensemble moderne au Québec (Couture 2013b). 
Dès les premiers concerts, la société présente, par exemple, la Symphonie d'instruments à vent (1920, rév. 1947) de Stravinsky, plusieurs œuvres de Webern, dont le Concerto pour neuf instruments, op. 24 (1934), Octandre (1923) de Varèse et Pierrot lunaire (1912) de Schoenberg, au côté d'œuvres récentes comme le suggère le programme de concert du 8 février 1973 (figure 1).

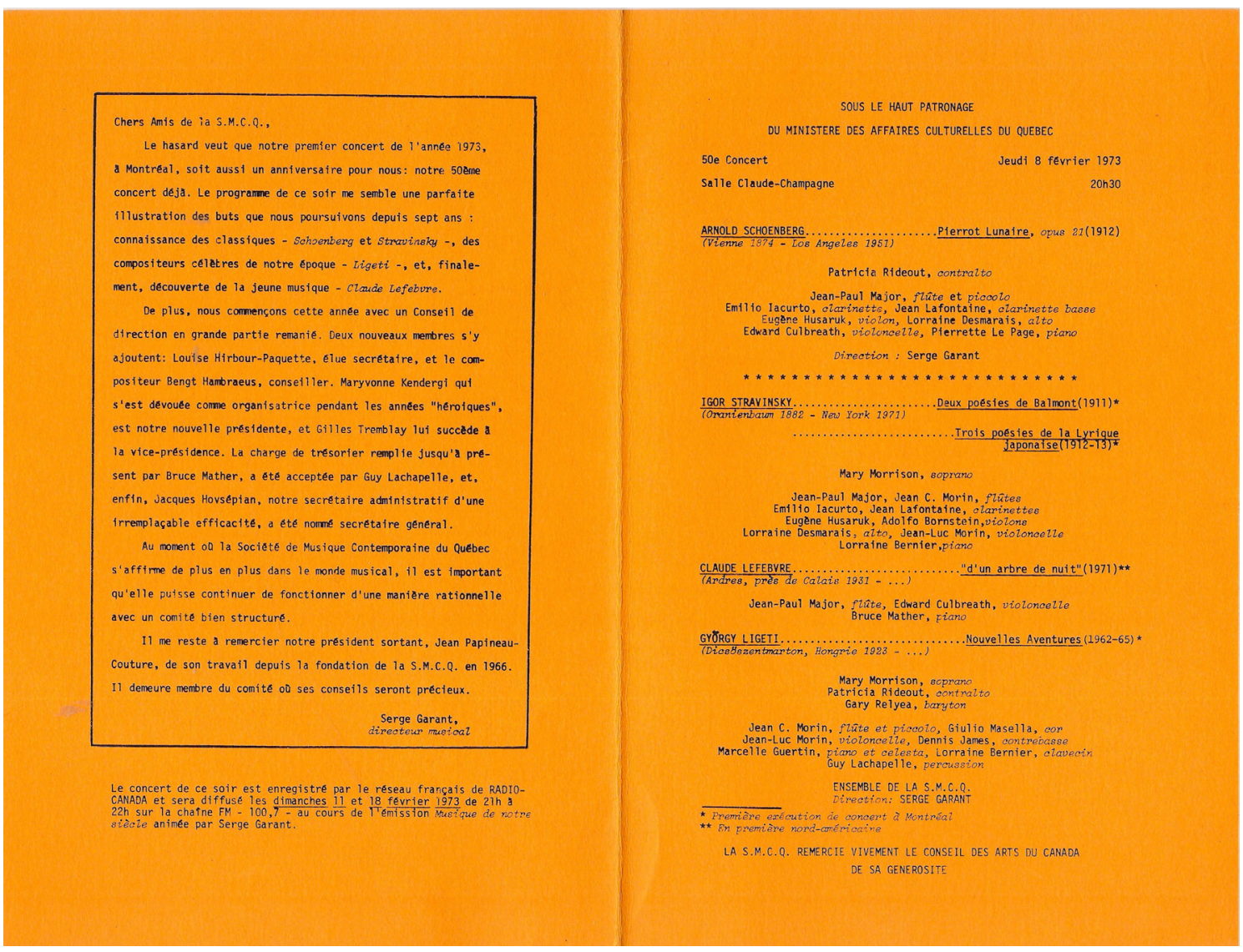

Figure 1: SMCQ, Programme du 50' concert, 8 février 1973, $3^{e}$ de couverture, $14 \mathrm{~cm} \times 21,5 \mathrm{~cm}$.

Une autre particularité de la programmation de la SMCQ est la présentation des œuvres dans une formule de concert thématique. Ainsi, pour pallier au sentiment de dispersion dans les œuvres sélectionnées, elles sont regroupées sous un même thème ${ }^{4}$. Il peut s'agir d'un médium comme l'acousmatique, d'un instrument comme les percussions, d'un interprète ou d'un ensemble - Louise Bessette, le Quatuor Morency - ou d'un même compositeur - Boulez, Murray Schafer, Vivier -, ou selon

4 En fait, cette formule de concert n'est pas réellement innovante puisque des organismes musicaux comme la Société Nationale de Musique et la Société de Musique Indépendante l'utilisaient déjà en France au début du xx siècle (Duchesneau 1997). Au Québec, Les Événements du neuf et l'Ensemble contemporain de Montréal font des concerts thématiques l'élément clef de la programmation des concerts (Couture 2013a). 
leur appartenance géographique (par exemple, le concert du 9 décembre 1993 est consacré à la musique italienne et les concerts du 19 janvier 1995 et du 23 janvier 1999 à la musique du Québec). Un autre moyen est de trouver un lien plus poétique entre les œuvres comme « Exoticanada », le 26 octobre 1995.

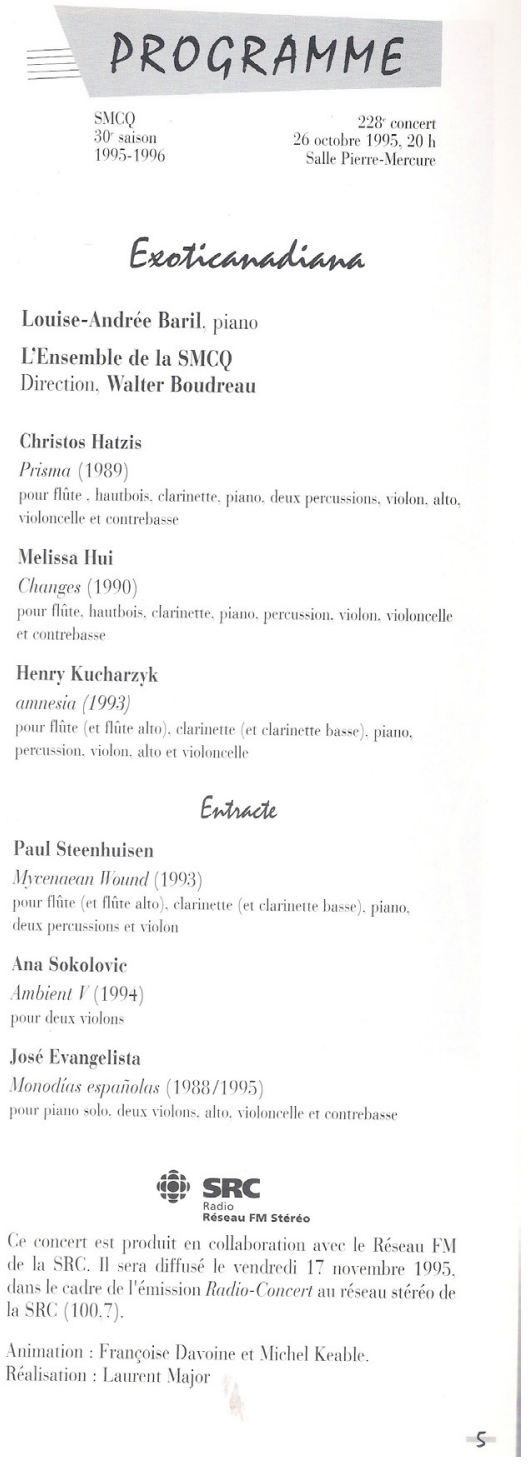

Figure 2: SMCQ, Programme du $228^{e}$ concert, 26 octobre 1995, p. 5, 10,7 cm x 27,7 cm.

Les programmes de concerts montrent le rôle de la SMCQ dans le développement du répertoire de musique contemporaine au Québec. Un modèle de présentation a rapidement été adopté, bien que le format des programmes ait évolué au fil du temps comme en témoigne la formule thématique introduite par Walter Boudreau autour des années 1990. La comparaison entre les programmes de différentes périodes permettra de discuter des efforts mis en œuvre par la SMCQ pour refléter sa pensée musicale jusque dans la conception visuelle de ses programmes. 


\section{LA PRÉSENTATION DES PROGRAMMES DE CONCERT DE LA SMCQ}

Dès le premier concert de la sMce, le 15 décembre 1966 à la salle ClaudeChampagne de l'Université de Montréal, des livrets de concert ont été distribués aux auditeurs présents afin de présenter les œuvres.

La page de couverture des programmes de salle a peu évolué entre les années $1967^{5}$ et 1979. S'y trouvent le nom et le logo de la société (figure 3).

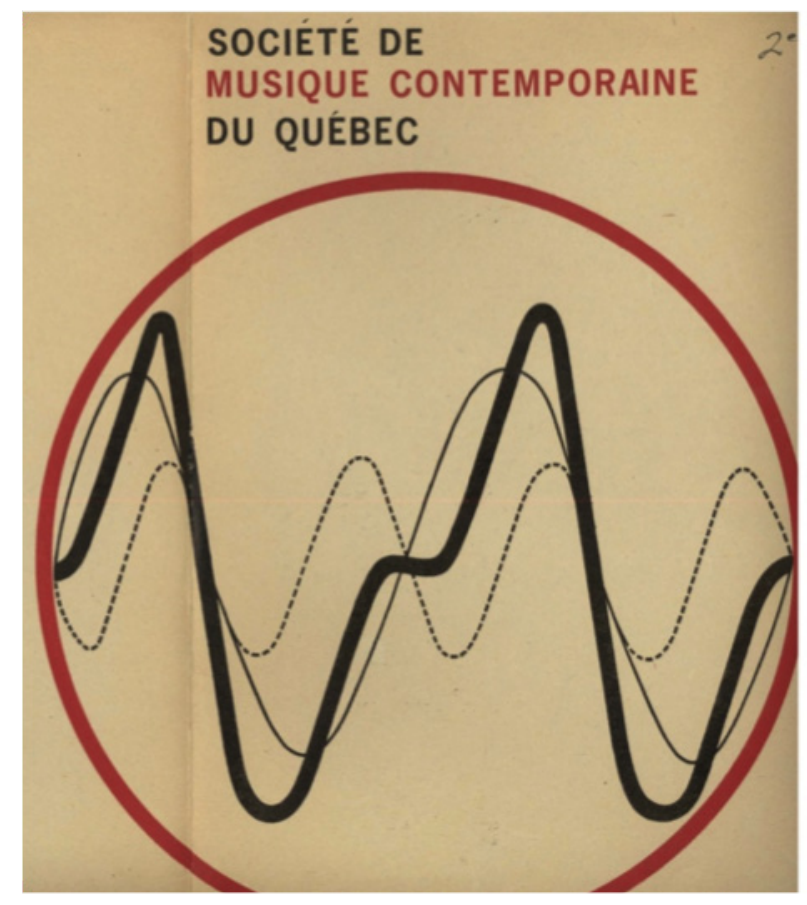

Figure 3 : SMCQ, Programme du $2^{e}$ concert, 16 février 1967, $1^{\text {re }}$ de couverture, $15,2 \mathrm{~cm} \times 23 \mathrm{~cm}$.

Le premier logo de la SMCQ est formé de deux ondes sinusoïdales et de leur résultante superposées dans un cercle. Cette image s'inspire du cadran d'un oscillateur et rappelle que, dans sa plus simple expression, le son est une onde. On pourrait lire ici une référence à la pureté moderniste d'un travail sur des composants élémentaires du son où la matière musicale est soumise à des techniques et des calculs sophistiqués contrôlés par l'œil du compositeur. Une lecture alternative de la figure de la modulation de fréquence amène un rapprochement avec la musique électroacoustique, et plus particulièrement avec le studio de musique électronique de Cologne, dont la sMce est un témoin des premiers développements au Québec. À cet égard, il importe de rappeler les liens privilégiés qu'entretenaient avec Stockhausen plusieurs membres du comité directeur de la SMCQ, notamment Serge Garant qui l'avait rencontré pendant

5 Le programme du concert du 15 décembre 1966 ne comporte pas de couverture. 
son séjour dans la classe d'analyse de Messiaen en 1951 et $1952^{6}$. En somme, ce logo suggère que la musique est une science dont on peut étudier l'histoire, la théorie, la pratique, mais également l'esthétique et les propriétés acoustiques. Dans un certain sens, la SMCQ invite à la découverte des musiques contemporaines ${ }^{7}$.

Entre 1979 et 1986, le comité directeur de la SMCQ opte pour une présentation plus sobre et le logo disparaît de la page de couverture (figure 4). Puis, en 1986, Anne-Marie Messier ${ }^{8}$ propose l'adoption d'un logo composé à partir de l'acronyme de l'organisme (figure 5). Ce logo sera en vigueur jusqu'en 2014.
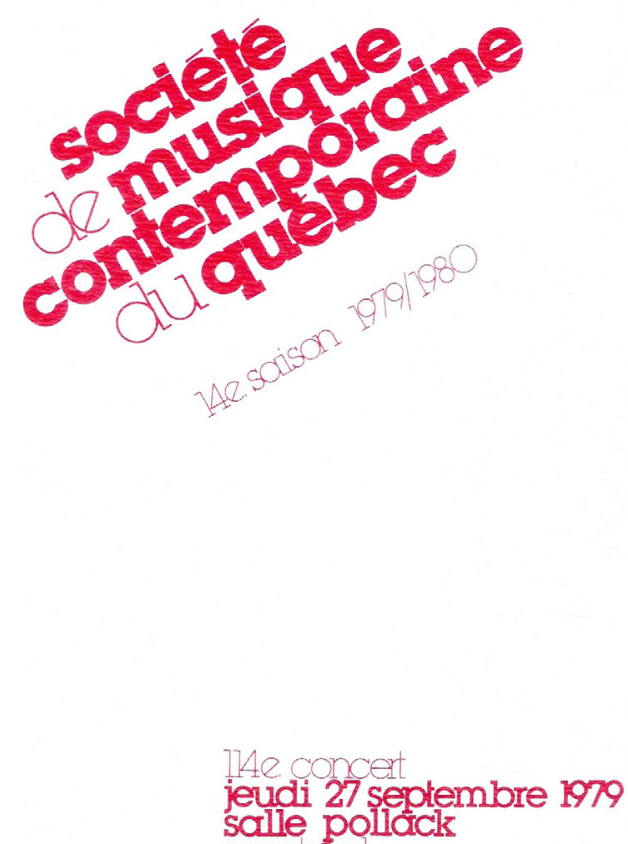

salle polack

Figure 4 : SMCQ, Programme du $114^{e}$ concert, 27 septembre 1979, $1^{\text {re }}$ de couverture, $21,4 \mathrm{~cm} \times 20,5 \mathrm{~cm}$.

6 Pour approfondir la réception de la pensée et de la musique de Stockhausen au Québec, les lecteurs se réfèreront au numéro consacré à cette question par la revue Circuit. Musiques contemporaines, vol. 19, $\mathrm{n}^{\circ} 2$, 2009.

$7 \quad$ Je remercie le lecteur anonyme pour ses remarques utiles qui m'ont permis de préciser certaines des idées avancées dans une première version au sujet du logo de la SMCQ.

8 Anne-Marie Messier occupe le poste de directrice administrative de la sMce de 1986 à 1996. 


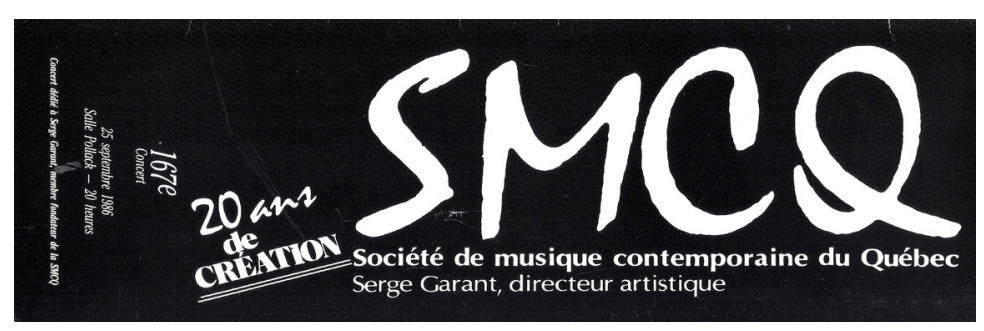

Figure 5 : smce, Programme du 167e concert, 25 septembre 1986, $1^{\text {re }}$ de couverture, $10,7 \mathrm{~cm} \times 27,7 \mathrm{~cm}$.

À partir de la quatrième saison (1969-1970), de plus en plus d'éléments se trouvent sur la couverture : le titre du concert (s'il y a lieu); l'année et le rang de la saison; le numéro de concert; la date et l'heure; le lieu. Considérant le programme de concert comme un outil de communication conçu par les organismes culturels pour informer les membres de l'auditoire, on constate que ces informations sont plus utiles à l'historien pour situer le contexte du concert qu'à l'auditeur déjà présent dans la salle.

À ces éléments de base, la direction de la SMCQ ajoute occasionnellement des slogans sur la page de couverture : "à l'œuvre depuis 1966 » est intégré dans le logo de la SMCQ sur les programmes de 1976 à 1979; "soyez absolument moderne » est inséré dans un bandeau supérieur des programmes de 1985 à 1986 (figure 6); « 20 ans de CRÉATION » figure sur les programmes de 1986 à 1987; remplacé par « 30 ans de concerts » en 1996-1997; puis « résolument contemporaine » en 2003-2004. Si certains de ces slogans sont de type informatif, d'autres portent une charge symbolique qu'il est opportun de commenter plus longuement.

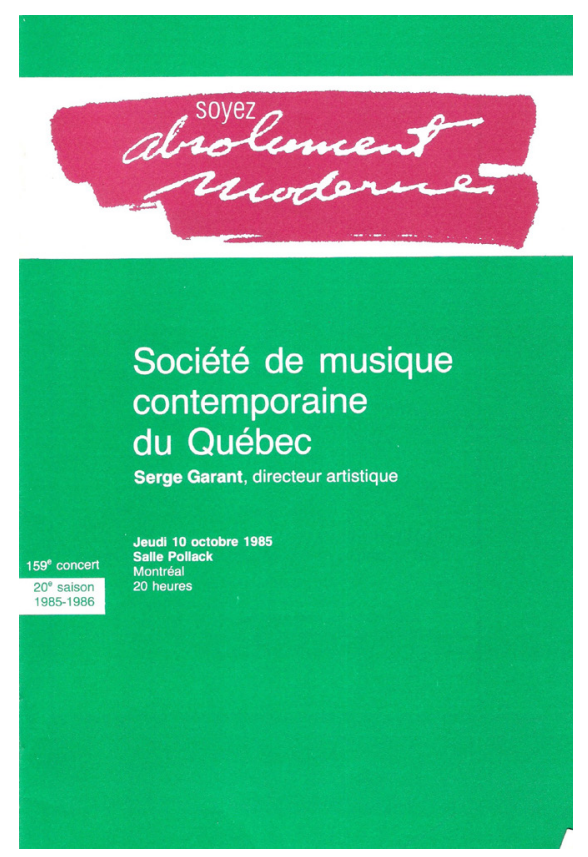

Figure 6: sMce, Programme du 159 concert, 10 octobre 1985, $1^{\text {re }}$ de couverture, 


\section{$13,7 \mathrm{~cm} \times 20,4 \mathrm{~cm}$}

En premier lieu, le slogan "Soyez absolument moderne " affiche une parenté indéniable avec le vers de Rimbaud : "Il faut être absolument moderne " (Une saison en enfer, "Adieu ", 1873), repris ensuite par les mouvements d'avant-garde9. Alain Bardel (2008) suggère de lire dans l'aphorisme rimbaldien un dénouement optimiste aux méditations anxieuses du narrateur du poème sur le salut de l'art et de l'humanité. Cette confiance en l'avenir repose cependant sur une condition (" il faut »): s'affranchir du passé. Par l'adjectif " moderne », Rimbaud en appelle ainsi à la notion de progrès. Mais il y a des limites à une acceptation aveugle de la nouveauté. Selon Bardel, Rimbaud a ajouté l'adverbe " absolument " "pour qu'on comprenne qu'il ne s'agit pas d'être en admiration béate devant le monde moderne tel qu'il est mais plutôt de se tenir aux aguets, ouvert à tout ce qui pourrait survenir de radicalement neuf et mériter le nom de progrès " (Bardel 2008). Dans le slogan de la SMCQ, cette idée d'ouverture attentive à la nouveauté (" absolument moderne ») est encore présente, mais un passage s'opère depuis la nécessité d'être (" il faut être ») de Rimbaud à l'impératif d'être («soyez»). Sous la forme d'un commandement, la sMce exhorte ses auditeurs ${ }^{10}$ à s'engager personnellement pour le futur de la musique.

En second lieu, les programmes de la saison 2003-2004 portent la mention " résolument contemporaine ». Ce slogan révèle moins un ordre adressé à un groupe de tiers qu'une affirmation identitaire, le sujet implicite étant la sMce. En d'autres mots, l'organisme se définit lui-même, d'une façon franche et décidée ("résolument»), comme un représentant de la musique " contemporaine ». L'expression " musique contemporaine ", quant à elle, renvoie, très largement, au répertoire de musique occidentale savante écrite après 1945 hors du système tonal, soit le noyau principal des œuvres diffusées par la SMCQ. En effet, la musique contemporaine, comprise dans son sens esthétique, réfère aux œuvres utilisant des systèmes d'organisation sonore tels le sérialisme, l'électroacoustique, ou la musique spectrale. La composition de la musique contemporaine présuppose donc une réflexion posée sur la musique en vue d'innover et de contribuer à son évolution historique (Deliège 2003). Ainsi, en se déclarant "résolument contemporaine », la sMce exprime fermement son identité musicale et son autorité par rapport aux œuvres contemporaines.

À compter de la $28^{\mathrm{e}}$ saison, 1993-1994, des images (photographies, dessins, illustrations) ornent ponctuellement la couverture des programmes de concert. De nature généralement conceptuelle (figure 7) ${ }^{11}$, les images de deux saisons se démarquent, car elles mettent à l'avant-plan le directeur artistique et musical de la sMce,

Walter Boudreau. À la $37^{\mathrm{e}}$ saison, on voit une photo en noir et blanc de Boudreau en

\footnotetext{
9 Que le lecteur anonyme d'une version antérieure de cet article soit remercié de cette précieuse observation.

10 L'utilisation de la deuxième personne du pluriel indique que l'organisme s'adresse à un groupe de personnes dont il s'exclut.

11 Escal observe une tendance à l'abstraction dans le cas des pochettes de disque de musique moderne et contemporaine (1996, p. 34).
} 
pleine action (figure 8). Pour la campagne de la $38^{\mathrm{e}}$ saison, on mise sur la marque de commerce de Boudreau : ses fameuses espadrilles rouges (figure 9).

Dans la société occidentale, les vedettes attirent le public dans les salles de concert, ce que défend la sociologue Nathalie Heinich dans son récent ouvrage De la visibilité (2012). Puisqu'en musique contemporaine les œuvres sont généralement peu connues du grand public, Walter Boudreau doit jouer ce rôle de tête d'affiche pour attirer de nouveaux auditeurs. La journaliste Dominique Olivier, dans un article pour 1'hebdomadaire montréalais Voir, offre un témoignage de l'attraction qu'exerce Boudreau :

L'homme aux souliers rouges, le chef très " in " de la SMCQ, devenu il y a deux ans son directeur artistique, fait couler beaucoup d'encre depuis. Walter Boudreau, une personnalité pour le moins extravertie, dynamique et même survoltée, donne une image médiatique captivante. Le voir entrer en courant et en sautant sur la scène, diriger avec une énergie peu commune et ensuite lancer force baisers dans la salle, à grands gestes ostentatoires, est une expérience visuelle qu'on a rarement l'occasion de faire au concert, où dominent habituellement le sérieux et l'austérité (Olivier 1990).

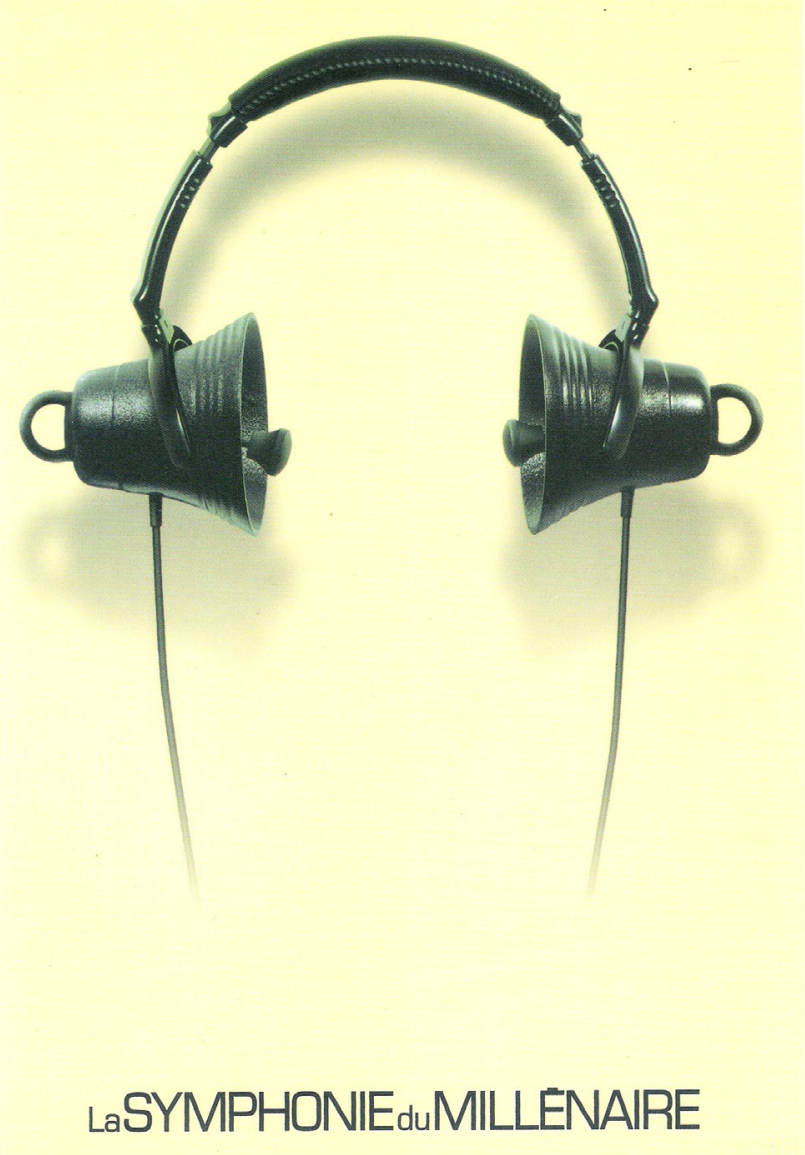

Figure 7: SMCQ, Programme de la Symphonie du millénaire, 3 juin 2000, $1^{\text {re }}$ de couverture, 
$15,1 \mathrm{~cm} \times 22,6 \mathrm{~cm}$, (C) Yvan Adam.

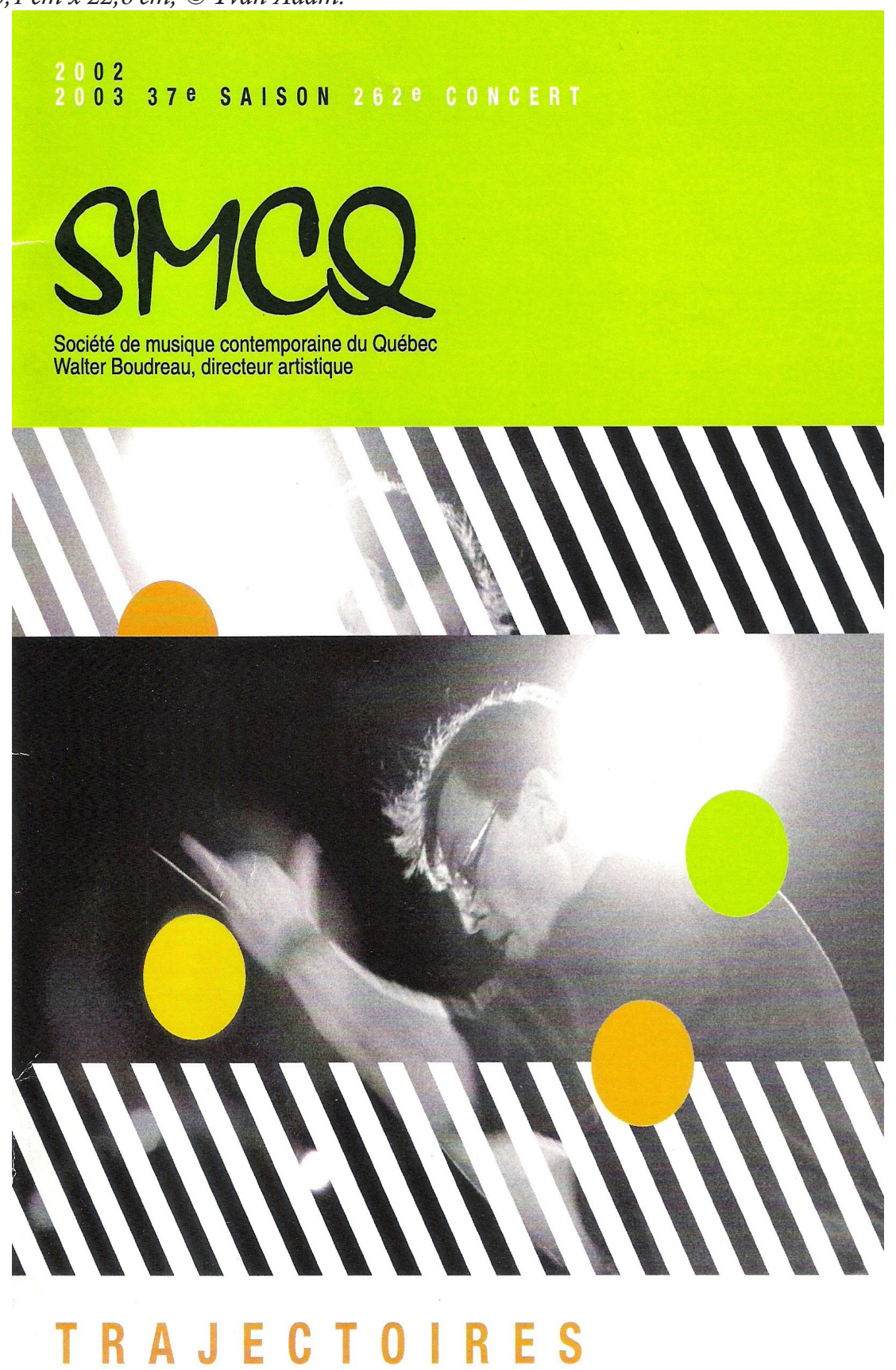

17 octobre $200220 \mathrm{~h}$

Salle Pierre-Mercure du Centre Pierre-Péladeau

Figure 8: SMCQ, Programme du 262 concert, 17 octobre 2002, $1^{\text {re }}$ de couverture, 13,7 cm x 19,1 cm. Graphisme : Elastik / Photo : Walter Boudreau, (C) Bruno Massenet. 


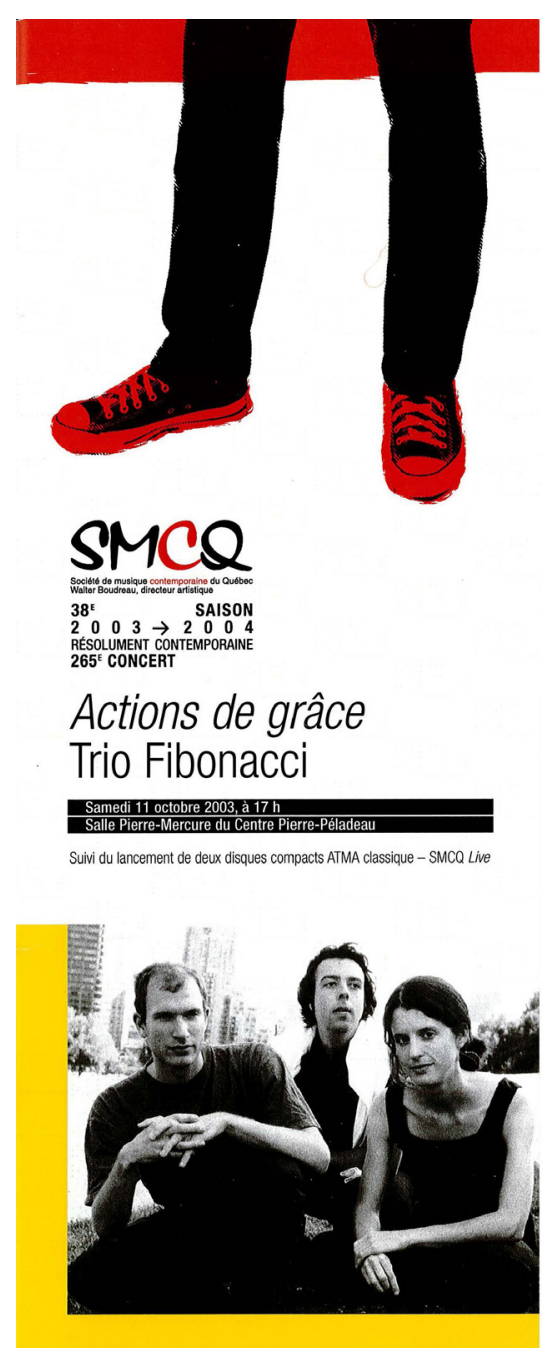

Figure 9 : sMce, Programme du $265^{e}$ concert, 11 octobre 2003, $1^{\text {re }}$ de couverture, $10,7 \mathrm{~cm} \times 27,7 \mathrm{~cm}$. Graphisme : Elastik / Photo : Trio Fibonacci (Gabriel Prynn, André Ristic, Julie-Anne Derome).

La notion de visibilité, qui s'apparente à celle de vedettariat, permet de mieux comprendre le choix de ces images. Walter Boudreau, en tant que directeur musical et artistique, est le principal ambassadeur de la SMCQ. Les représentations des œuvres que l'organisme défend sont véhiculées à travers son image. Ainsi, comme le souligne Escal, à propos des illustrations des pochettes de disques, ces images forment un paratexte des œuvres interprétées en concert parce qu'elles préparent et structurent la réception des auditeurs (1996, p. 34).

La sociologie des textes fournit à la présente analyse un autre ensemble de paratextes non verbaux. Ainsi, McKenzie défend la théorie selon laquelle les formes physiques à travers lesquelles les messages sont transmis (format, découpage en sections, mise en 
page, typographie, etc.) affectent le processus de production du sens de l'œuvre chez le récepteur (1991, p. 25-54).

À la SMCQ, il est intéressant de noter que la morphologie des programmes a changé plusieurs fois au fil des ans. Il y a eu le format livret, c'est-à-dire une feuille de format lettre pliée en deux sur le sens de la largeur (de 1965 à 1978, de 1982 à 1986, de 2009 à aujourd'hui; voir figure 6), le format carré (de 1978 à 1982; figure 4), et le format rectangulaire, c'est-à-dire une feuille de format lettre pliée en deux sur le sens de la longueur (de 1986 à 2009; voir figure 9). Les formats livret et rectangulaire ont été les plus populaires, car ils utilisent un format de papier standard, ce qui permet des économies en terme de papeterie et d'impression. Le format rectangulaire est sans doute une initiative d'Anne-Marie Messier, directrice administrative de 1986 à 1996, et co-propriétaire avec son frère de la coopérative de recherche et de production en communication Les Nuages. Le programme rectangulaire a connu une certaine longévité, même s'il paraît surdimensionné de prime abord. Il est long et étroit, mais facile à tenir en main et à consulter. Surtout, il se démarque des formats de programme usuels, suggérant par-là que la SMCQ occupe une position particulière dans le milieu de la musique contemporaine.

Enfin, on retrouve à l'intérieur du programme d'autres messages paratextuels situant les œuvres. Ceux-ci sont disposés dans un ordre précis, la configuration des sections ayant peu changé au fil du temps :

1. un mot de présentation rédigé par un des membres de l'équipe de la $\mathrm{SMCQ}^{12}$;

2. une page de présentation du déroulement du concert (titre, coordonnées, interprètes, compositeurs, titre de l'œuvre, effectif musical et minutage, partenaires; voir figures 1,2 et 10);

3. les notices des œuvres (titre, nom du compositeur ainsi que ses dates et lieux de naissance et de décès, date et lieu de la première audition, description);

4. les notices biographiques des compositeurs;

5. la liste des interprètes;

6. les coordonnées et la présentation de l'équipe de la SMCQ, de l'équipe de production du concert, des bénévoles et des partenaires;

7. 1'annonce du prochain concert de la SMCQ (à partir de la $12^{\mathrm{e}}$ saison);

8. les annonces des commanditaires et des partenaires (à partir de la $13^{\mathrm{e}}$ saison).

12 Maryvonne Kendergi, vice-présidente et organisatrice de la SMCQ, a longtemps réalisé cette tâche avec la participation occasionnelle de Serge Garant, directeur artistique de 1966 à son décès en 1986, ou encore de Jean Papineau-Couture, président de 1966 à 1972. C'est aujourd'hui Walter Boudreau, directeur artistique depuis 1988 qui signe les textes de présentation des concerts et, s'il y a lieu, un représentant des organismes partenaires. 


\section{VERS UNE ÉTOILE}

6 DÉCEMBRE 2009, 16 H | ÉGLISE DE L'MMACULEEE CONCEPTION

| ||||||||||||||||||||||||||||||||||||||||||||||||||||||||||||||||||||||||||||

AU PROGRAMME

Jean Lesage, Preludio quasi una fantasia (création) 4'30

Jean-Willy Kunz, orgue

Gilles Tremblay, Vers une étoile (Compostelle II) (1993)

Jean-Willy Kunz, orgue

Pierre Saint-Amand, scénographie

Yves Daoust, Chorals ornés (2007-2008) 45

Musique électroacoustique avec orgue

Régis Rousseau, orgue
Yves Daoust, diffusion

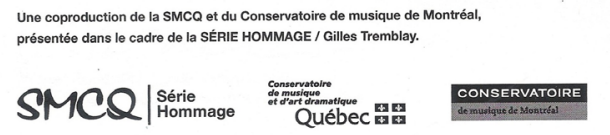

Figure 10: SMCQ, Programme de concert, 6 décembre 2009, 1ère de couverture, $14 \mathrm{~cm} \times 21,5 \mathrm{~cm}$.

À propos des éléments susmentionnés, il est nécessaire de préciser que les biographies des compositeurs ont fait leur apparition dans les programmes de concert de la SMCQ seulement à compter de la $17^{\mathrm{e}}$ saison (1982-1983). Auparavant, seules les dates et les lieux de naissance et de mort des compositeurs étaient mentionnés et intégrés dans la section des œuvres. De plus, les compositeurs sont généralement présentés après les œuvres puisqu'à la SMCQ, le souci d'établir un répertoire de musique contemporaine au Québec motive la décision de mettre les œuvres à l'avant-plan ${ }^{13}$. Toutefois, dans le cas d'un concert hommage, la sMce publie un programme de concert spécifique où l'attention est orientée vers le compositeur. En plus des éléments habituels, on retrouve dans ce type de programmes des témoignages, des extraits des écrits du compositeur célébré, des critiques de concerts, des extraits de partitions, la liste des œuvres du compositeur, etc.

Quant à la section dédiée aux interprètes, elle emprunte d'abord l'apparence d'une liste où l'on nomme le directeur artistique, les solistes et les musiciens engagés par

13 Nicolas Donin constate une pratique inverse dans le cas des programmes de salle de Domaine musical puisque les compositeurs étaient présentés avant les œuvres (2011, p. 41). 
la SMCQ. À la $17^{\mathrm{e}}$ saison (1982-1983), les noms du directeur artistique et des solistes sont accompagnés d'une courte notice biographique et d'une photo (à partir de la 34 saison, 2004-2005). Ces changements dans le contenu des programmes témoignent de la place de plus en plus importante attribuée aux interprètes.

\section{LES NOTICES DES CEUVRES}

La comparaison des notices de quelques œuvres présentées à la SMCQ entre 1966 et 2013 permettra de dégager les principales caractéristiques de ces paratextes tout en discutant du sens induit par la SMCQ. Les œuvres choisies figurent parmi les plus jouées en concert par l'organisme : Intégrales (1924-1925) d'Edgard Varèse jouée 7 fois, les Variationen, op. 27 (1936) d'Anton Webern jouées 4 fois, ... le sifflement des vents porteurs de l'amour... (1971) de Gilles Tremblay interprétées 19 fois et Zipangu (1977) de Claude Vivier présentées 5 fois en concert. Cette sélection offre la possibilité de vérifier si les notes de programmes d'une ouvre sont rééditées ou demeurent les mêmes à chaque reprise et de comprendre les raisons et les effets de ces pratiques.

En premier lieu, l'analyse des textes révèle qu'ils sont majoritairement rédigés par les compositeurs eux-mêmes, plutôt que par un musicologue, un critique musical ou une personne issue de la SMCQ. À cet égard, Nicholas Cook rappelle que : « Le concept de musique comme matière première donne naturellement au compositeur une position centrale : il est à l'origine du produit » (Cook 2006, p. 32) ${ }^{14}$. Le compositeur fait donc figure d'autorité en musique et ses écrits sont considérés comme une source d'information privilégiée sur une œuvre. Ce sont donc vers eux que la SMce se tourne principalement pour rédiger ou fournir les notes de concert sur leur propre œuvre. Les exemples suivants présentent des particularités d'écriture des notices des œuvres.

Signé par le compositeur Gilles Tremblay, le paratexte accompagnant ... le sifflement des vents porteurs de l'amour... (figure 11), bien que succinct, éclaire l'auditeur sur la signification du titre de l'œuvre (" une citation du Cantique spirituel XIV de Saint Jean de la Croix »), fournie des pistes d'écoute (il y a deux étapes : "gels, pureté éclatante du silence, souffle, franges d'existence "; et " chaleur qui fond et fertilise dans des possibilités multipliées et sans fin »), évoque ses sources d'inspiration (" le Resurrexit et l'Alleluia de la messe de Pâques en plain-chant »), rappelle le processus de création (« les séances d'expérimentation à la flûte furent extrêmement utiles et stimulantes, surtout en ce qui concerne l'investigation des "whistle tones » ou harmoniques d'embouchure ") et nomme les dédicataires de l'œuvre (la mère du compositeur, Robert Cram et Ian Bernard). Cette note est également révélatrice de l'approche poétique du compositeur, connu pour la richesse de son vocabulaire et pour décrire les œuvres par des " concepts évocateurs d'images sonores " (Lefebvre 1995 , p. 6) ${ }^{15}$.

14 "The concept of music being a kind of commodity naturally gives the composer a position of centrality, as the generator of the core product "(Cook 2000, p. 24).

15 Les lecteurs pourront constater d'eux-mêmes l'originalité de la pensée de Gilles Tremblay en se référant aux deux numéros de la revue Circuit. Musiques contemporaines (vol. 5, no 1, 1994 et vol. 6, n ${ }^{\circ} 1$, 1995) consacrés aux écrits du compositeur, sous la direction de Marie-Thérèse Lefebvre. 


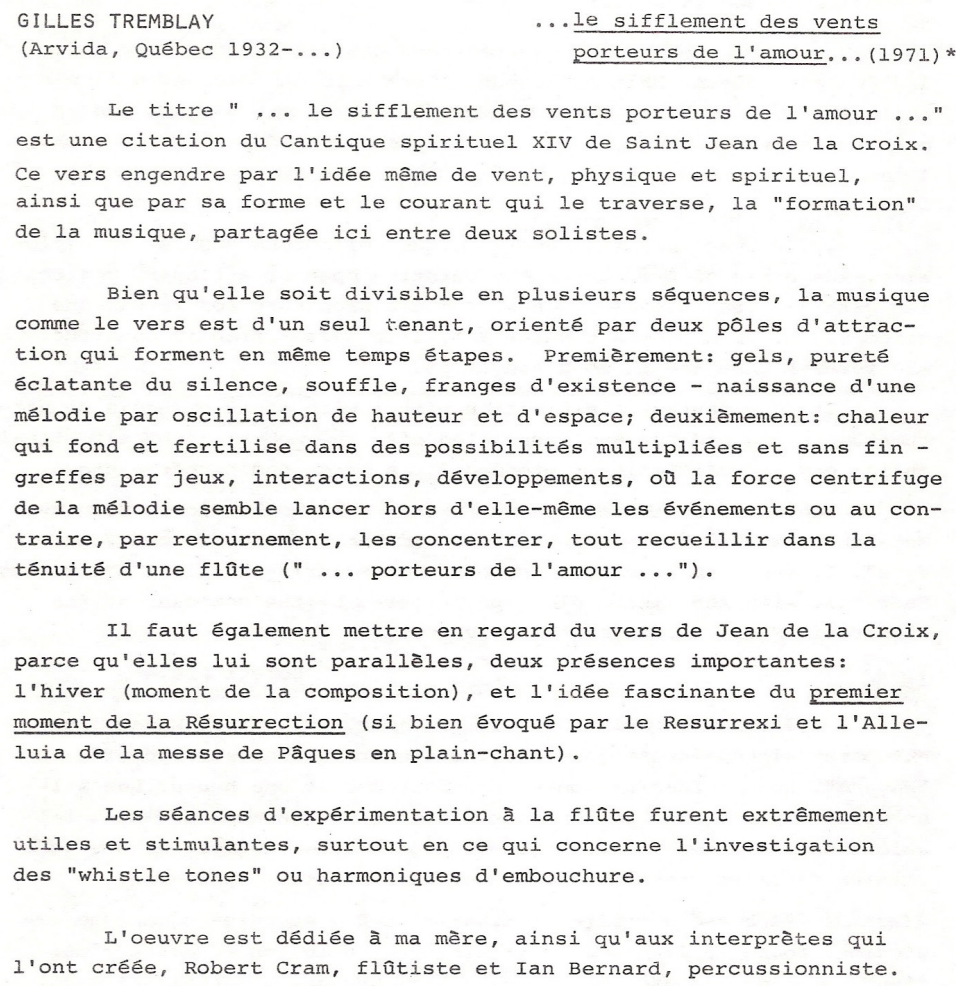

Gilles Tremblay

GILLES TREMBLAY sera l'invité des Musialogues, une activité de la Faculté de musique et du Service d'animation de I'Université de Montréal, les 13 et 14 décembre 1972, à $20 \mathrm{hoO}$, au Centre communautaire de I'U.M., 2332, boul. Edouard-Montpetit. Au cours de la première soirée, le compositeur évoquera les principaux aspects de sa carrière. Le lendemain, il parlera de son récent voyage en Extrême-Orient d'où il rapporte des docinents sonores exceptionnels.

* Première montréalaise.

Figure 11: sMce, Programme du 47e concert, 7 décembre 1972, p. 6, $14 \mathrm{~cm} \times 21,5 \mathrm{~cm}$.

Dans le cas d'Intégrales d'Edgard Varèse, la notice de l'œuvre publiée par la SMCQ est une citation issue de son ouvrage Le poème électronique Le Corbusier (1959, p. 192) expliquant sommairement la relation en musique des intégrales dans l'espace (figure 12). 
Les Intégrales furent conçues pour une projection spatiale. Je les construisis pour certains moyens acoustiques qui n'existaient pas encore, mais qui, je le savais, pouvaient être réalisés et seraient utilisés tôt ou tard.

... Tandis que dans notre système inusical nous répartissons des quantitês dont les valeurs sont fixes, dans la réalisation que je souhaitais, les valeurs auraient continuellement changé en relation avec une constante. En d'autres termes, ç'aurait été cornme une sêrie de variations, les changements résultant de légères altêrations de la forme d'une fonction ou de la transposition d'une fonction à une autre. Pour mieux me faire comprendre - car l'oeil est plus rapide et plus discipliné que l'oreille transférons cette conception dans le monde visuel et considērons la projection changeante d'une figure géométrique sur un plan, avec la figure et le plan qui tous deux se meuvent dans l'espace, mais chacun avec ses propres vitesses, changeantes et variēes, de translation et de rotation. La forme instantanée de la projection est déterminée par l'orientation relative entre la figure et le plan à ce moment. Mais en permettant à la figure et au plan d'avoir leurs propres mouvements, on est capable de reprësenter avec la projection une image hautement complexe apparemment irilprêvisible; de plus, ces qualités peuvent être augmentées ultérieurement en laissant la forme de la figure géométrique varier aussi bien que ses vitesses...

Edgard Varèse

Ecuatorial (1934)

pour voix de basse, cuivres, instruments électroniques, percussion

\begin{abstract}
Le texte d'Ecuatorial est extrait du livre sacré des Maya-Uuiché (du Guatemala) le Popul Vuh; c'est l'invocation de la tribu perdue dans les montagnes, après avoir quittê la "cité de l'abondance". Le titre suggère simplement les régions où fleurissait l'art précolombien. Je voulais donner à la musique la même intensité rude, élēmentaire, qui caractêrise ces oeuvres étranges et primitives. L'exêcution devrait être dramatique et incantatoire, guidée par la ferveur implorante du texte, et suivant les inaicationsdynamiques de la partition.
\end{abstract}

Edgard Varèse

Figure 12 : sMce, Programme du 72 concert, 6 novembre 1975, p. 6, 17,7 cm x $21,6 \mathrm{~cm}$.

Quant à elle, la notice des Variationen, op. 27 de Webern a évoluée au fil du temps, entre autre parce qu'elle n'est pas rédigée par le compositeur. Ces différentes notices incluent généralement un extrait de correspondance de Webern avec la poétesse Hildegarde Jones, comme dans la note de programme préparée par Louise Hirbour-Paquette pour le concert du 4 mai 1978 (figure 13), où le compositeur rend compte de l'avancement de l'écriture de l'œuvre et exprime l'espoir d'arriver à un aboutissement : 
(RE)PRÉSENTER LES EUVRES MUSICALES.

L'EXEMPLE DES PROGRAMMES DE CONCERT DE LA SOCIÉTÉ DE MUSIQUE CONTEMPORAINE DU QUÉBEC

ANTON WEBERN

Vienne, 1883 - Mittersizl, 1945

Variations pour piano

op. 27 (1936)

\footnotetext{
Très souvent dans les écrits de Webern revient cette phrase de Goethe sur les premières plantes du monde : La racine n'est pas différente de la tige, Za tige de la feuizze et la feuizie de za fleur: ce sont des variations d'une même idée.

Les Variations op. 27 semblent être un exemple parfait de cette idée d'unicité. Il ne s'agit pas en effet d'un travail de paraphrase sur un thème déterminé. Le mot variation est ici $1^{\prime}$ expression d'une construction à partir d'un nombre minimum d'éléments constamment présents sous un aspect nouveau.

L'oeuvre est en trois mouvements : Très modéré; Très vite;

souple et calme.
}

Louise Hirbour-Paquette

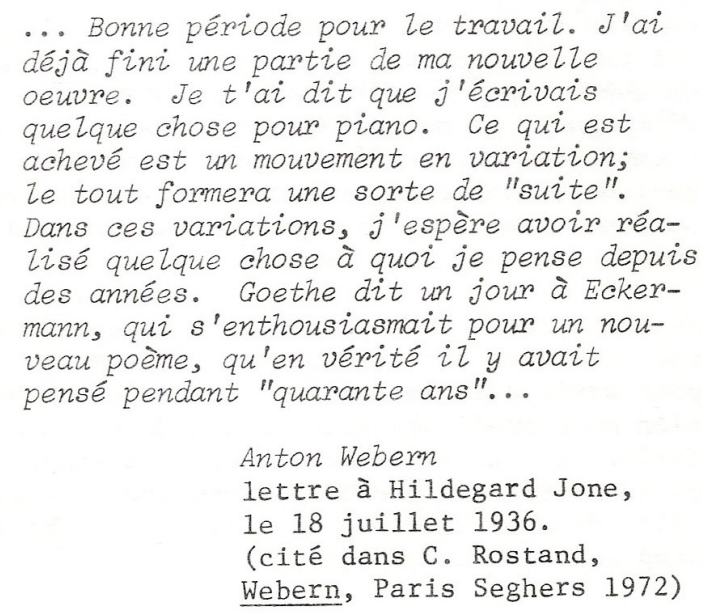

Figure 13 : sMce, Programme du 107e concert, 4 mai 1978, p. 4, 13,8 cm x 21,7 cm.

Cette citation laisse entendre que cette pièce, sa seule œuvre pour piano avec un numéro d'opus, occupe une place centrale dans la production wébérienne. Les notes sur Zipangu de Vivier ont également été réécrites plusieurs fois. Lors du concert du 26 avril 1984, Vivier explique :

Autour d'une mélodie, j'explore, dans cette œuvre, différents aspects de la « couleur ». J'ai tenté de " brouiller » mes structures harmoniques par 
l'emploi de différentes techniques d'archet. Ainsi s'opposent un bruit coloré obtenu par pression exagérée de l'archet sur les cordes et les harmoniques pures, lorsqu'on revient à la technique normale.

Les notes du concert du 9 février 1995 sont signées par Serge Garant, compositeur décédé en 1986, ami de Vivier et premier directeur artistique de la SMCQ. Garant cite d'abord la notice écrite par Vivier, puis ajoute des informations supplémentaires afin de guider l'écoute des auditeurs :

La mélodie, dont parle le compositeur, est ici toujours présente. Au début et à la fin elle est clairement énoncée mais dans le reste de l'œuvre, elle subit toutes sortes de transformations.

Au final, pour les concerts des 7 mars 2008, 28 mai 2012 et 21 février 2013, c'est la musicologue Martine Rhéaume qui rédige la note. Rhéaume renvoie également à la notice de Vivier et insiste sur le timbre qui, plus que la mélodie, est au cœur de l'œuvre prouvant ainsi sa connaissance approfondie de l'œuvre :

Il devient clair à l'écoute que ces façons variées d'aborder les cordes créent l'intérêt de cette œuvre. On y entend la même mélodie sous la forme d'un dialogue entre cordes aiguës - d'où se détache parfois un violon solo - et cordes graves. Les cordes y sont en alternance très chantantes et très sombres; on trouve dans le grave une impression sinistre, et dans l'aigu, volontairement grinçante. S'insèrent des points de repère parfois légers et sautillants, parfois brusques et violents, haletants (Rhéaume, Programme du concert du 21 février 2013).

Alors que l'œuvre musicale, dans son identité idéale, est relativement stable (Escal 1996, p. 294; Genette 1987, p. 374), " son interprétation et son écoute 1[a] renouvellent sans cesse, 1[a] même ne revenant jamais que pour apporter du différent, ce qui fait qu'aucune histoire ne l'épuise " (Escal 1996, p. 294). Or la comparaison des notices démontre qu'il n'y a pas eu de changements significatifs dans les descriptions des œuvres présentées par la SMCQ. Depuis la fondation de l'organisme, les responsables des communications réutilisent fréquemment les mêmes notes, surtout si elles émanent de la main du compositeur lui-même. N'y a-t-il pas eu, en plus de quarante ans d'histoire, de nouvelles analyses, de nouveaux comptes rendus de ces œuvres? La réception des œuvres est-elle figée? Répondant à cette question par la négative, Genette et Escal insistent sur le caractère fonctionnel du paratexte :

Étant immuable, le texte est par lui-même incapable de s'adapter aux modifications de son public, dans l'espace et dans le temps. Plus flexible, plus versatile, toujours transitoire parce que transitif, le paratexte lui est en sorte un instrument d'adaptation : d'où ces modifications constantes de la " présentation » du texte (c'est-à-dire de son mode de présence au monde), $d u$ vivant de l'auteur par ses propres soins, puis à la charge bien ou mal assumée, de ses éditeurs posthumes (Genette 1987, p. 375). 
Or les messages qui accompagnent [1'œuvre] à chaque publication, éphémères, se renouvellent et s'annulent sans cesse. Leur temporalité s'emballe, en quelque sorte. C'est qu'ils ont pour fonction de gérer hic et nunc le rapport de l'œuvre à son récepteur, réel ou putatif (Escal 1996, p. 294).

Il y aurait donc lieu de penser qu'en reprenant les mêmes notes, les organisateurs de la sMce tendent à négliger la réalité du public actuel. Néanmoins, on constate depuis quelques années un changement de la conduite éditoriale de la SMCQ. En effet, les programmes de concert des récentes séries rendant hommage à Vivier en 2008, Tremblay en 2010, puis Sokolovic en 2012, et Gougeon en 2014, ont été 1'occasion de rédiger de nouvelles notes de programme à partir de sources variées : les écrits des compositeurs, certes, mais également des extraits de journaux témoignant de la réception à la création des œuvres, des témoignages, des extraits d'analyse ou des nouveaux textes rédigés par des musicologues.

Dans la perspective d'une révision des descriptions des œuvres, afin de les mettre à jour par rapport aux développements récents de l'histoire de la musique (par exemple, en situant les œuvres dans l'ensemble de la carrière des compositeurs), les responsables de la SMCQ devront tenir compte de la morphologie, de l'écoute et des attentes du public. Jean-Simon Robert-Ouimet présente une réflexion intéressante en ce sens dans le cadre de son mémoire de maîtrise (2012) que la SMCQ pourrait poursuivre et, dans une étape ultérieure, tester auprès de son public.

\section{CONCLUSION}

Dans la lignée de la proposition d'une historiographie critique de la création musicale après 1945 d'Anne-Sylvie Barthel-Calvet, qui s'appuie sur cette multitude d'imprimés « qui excèdent les seules archives proprement dites d'un compositeur ou d'une institution; elle intègre et leur confronte des documents publiés (textes, programmes, etc.) plus ou moins spécifiques ou banaux, excluant de ce fait de récuser telle ou telle source documentaire " (Barthel-Calvet 2011, p. 11), le programme de concert constitue une mine d'informations originale sur les œuvres. À la base, le programme de concert (ou livret) est « édité pour accompagner le concert et a pour but de préciser ce qui va être joué. Il donne des informations plus ou moins développées sur les auteurs, les œuvres, les interprètes " (Lacombe 2009, p. 59). Dans le cas de la SMCQ, en plus des notes sur les œuvres, les textes de présentation des concerts rédigés par le directeur artistique ou des membres du conseil de direction, révèlent d'abondants détails sur le contexte de production et de diffusion des concerts, ce qui justifie l'analyse de ces objets à titre de paratextes de l'œuvre musicale.

Mis en réseau avec d'autres documents, le programme de concert permet une nouvelle lecture de l'œuvre menant soit à une explication de sa genèse, de son texte ou encore de sa réception. Cependant, ce document imprimé ne se limite pas à une collection de commentaires informatifs sur la musique qui sera interprétée lors du concert. Il comporte également des éléments significatifs d'analyse du fonctionnement de l'organisme (la composition des directions artistique et administrative, les partenaires, la liste des bénévoles, etc.), des choix esthétiques opérés par l'organisme, 
de l'organisation de la programmation musicale dans le cadre d'un concert (voir d'une saison de concerts), de la logique sous-tendant la présentation de la programmation musicale dans le programme de concert. En d'autres mots, ce type de document s'avère un précieux outil de réflexion historiographique pour les chercheurs en musique. Il est donc pertinent de réfléchir sur sa fabrication et son contenu afin de contribuer au développement de l'étude des sociétés de concerts.

\section{BIBLIOGRAPHIE}

Jesus Aguila, Jesus (1992), Le domaine musical. Pierre Boulez et vingt ans de création contemporaine, Paris, Fayard.

Bardel, Alain (2008), " "Adieu" ou l'impossible adieu ", http://abardel.free.fr/petite_anthologie/ adieu_commentaire.htm, consulté le 17 février 2015.

Bashford, Christina (2003), " Not just "G.". Towards a History of the Programme Note ", dans Michael Musgrave (dir.), George Grove, Music and Victorian Culture, New York, Palgrave Macmillan, p. 115-142.

Bent, Ian (dir.) (1994), Music Analysis in the Nineteenth Century, "2. Hermeneutic Approaches", Cambridge, Cambridge University Press.

Botstein, Leon (1992), "Listening through Reading. Musical Literacy and the Concert Audience ", $19^{\text {th }}$-Century Music, vol. XVI, no 2, p. 129-145.

Campos, Rémy, et Nicolas Donin (2005), « La musicographie à l'œuvre. Écriture du guide d'écoute et autorité de l'analyste à la fin du XIX ${ }^{\mathrm{e}}$ siècle », Acta Musicologica, vol. LXXVII, nº 2, p. 151-204.

Cook, Nicholas ([1998]2000), Music. A Very Short Introduction, Oxford, Oxford University Press. [Traduction française : Musique, une très brève introduction, Paris, Allia, 2006.]

Couture, Ariane (2013a), "Institutions et création musicale à Montréal de 1966 à 2006. Histoire et orientations artistiques de la SMCQ, des Événements du Neuf, de l'ECM et du NEM », Thèse de doctorat, Université de Montréal.

Couture, Ariane (2013b), "Parcours dans la programmation des concerts du Nouvel Ensemble Moderne », Circuit. Musiques contemporaines, vol. 23, n 3, p. 63-73.

Dale, Catherine (2003), "The Analytical Concert Programme Note. Its Growth and Influence in Nineteenth-Century Britain ", Music Analysis in Britain in the Nineteenth and Early Twentieth Centuries, London, Ashgate.

Dal Molin, Paolo (2011), «Le répertoire des programmes de concert en France après 1945 : remarques sur la méthode et les enjeux. Notes de travail sur les Rencontres internationales de musique contemporaine (Metz, 1972-1991) ", dans Anne-Sylvie Barthel-Calvet (dir.), Propositions pour une historiographie critique de la création musicale après 1945, Metz, Centre de recherche universitaire lorrain d'histoire, p. 51-78.

Deliège, Célestin (2003), Cinquante ans de modernité musicale : de Darmstadt à l'Ircam. Contribution historiographique à une musicologie critique, Sprimont, Mardaga.

Donin, Nicolas (2011), « Pour une analyse des documents d'accompagnement du concert. L'exemple des programmes de salle du Domaine musical », dans Anne-Sylvie Barthel-Calvet (dir.), Propositions pour une historiographie critique de la création musicale après 1945, Metz, Centre de recherche universitaire lorrain d'histoire, p. 31-49.

Duchesneau, Michel (1997), L'avant-garde musicale et ses sociétés à Paris de 1871 à 1939, Sprimont, Mardaga.

Enthoven, Gabrielle ([1951]1967), " Playbill, Programme », dans Phyllis Hartnoll (dir.), Oxford Companion to the Theatre, London, Oxford University Press, p. 742-744. 
Escal, Françoise (1996), Aléas de l'œuvre musicale, Paris, Hermann.

Fuld, James J. (1980-81), «Music Programs and Posters. The Need for an Inventory », Notes, vol. XxxvII, p. 520-532.

Garant, Serge (1967), «Mot de présentation », Programme du troisième concert de la sMce, 5 avril 1967, p. 1.

Genette, Gérard (1987), Seuil, Paris, Seuil.

Heinich, Nathalie (2012), De la visibilité. Excellence et singularité en régime médiatique, Paris, Gallimard.

Hibberd, Sarah (2002), "Programme, Programme notes ", dans Alison Latham (dir.), The Oxford Companion to Music, Oxford, Oxford University Press, www.oxfordmusiconline.com, consulté le 19 novembre 2012.

Lacombe, Hervé (2009), « Conception et méthode du RPCF (Répertoire des programmes de concert en France) ", Bulletin de l'AIBM Groupe français, n 17, p. 59-64, www.rpcf-projet.fr/archives/526, consulté le 10 juillet 2014.

Lefebvre, Marie-Thérèse (1994), «Éditorial », Circuit. Musiques contemporaines, vol. 5, nº 1, p. 5-7.

Richard Macnutt, Richard (2002), " Programme [program] ", dans Alison Latham (dir.), Oxford Music Online, Oxford, Oxford University Press, www.oxfordmusiconline.com, consulté le 19 novembre 2012.

McKenzie, Donald F. (1991), La bibliographie et la sociologie des textes, Paris, Éditions du Cercle de la Librairie.

Olivier, Dominique (1990), «Walter Boudreau - L'homme-orchestre », Voir, Édition du 30 août au 5 septembre 1990.

Pasler, Jann (2007), « Concert Programs and their Narratives as Emblems of Ideology », Writing through Music. Essays on Music, Culture, and Politics, New York, Oxford University Press, p. 365-416.

Robert-Ouimet, Jean-Simon (2012), « La médiation musicale. Le cas de la note de programme et de l'intervention orale », Mémoire de maîtrise, Université de Montréal.

Simeone, Nigel (2001), "Programme note ", dans Laura Williams Macy (dir.), Grove Music Online, www.oxfordmusiconline.com, consulté le 19 novembre 2012.

Société de musique contemporaine du Québec (1965-2013), programmes de concert, archives personnelles de l'auteure. 